\title{
Reading behavior predicts syntactic categories
}

\author{
Maria Barrett and Anders Søgaard \\ University of Copenhagen \\ Njalsgade 140 \\ DK-2300 Copenhagen $\mathrm{S}$ \\ $\{d t q 912$, soegaard $\}$ ahum.ku.dk
}

\begin{abstract}
It is well-known that readers are less likely to fixate their gaze on closed class syntactic categories such as prepositions and pronouns. This paper investigates to what extent the syntactic category of a word in context can be predicted from gaze features obtained using eye-tracking equipment. If syntax can be reliably predicted from eye movements of readers, it can speed up linguistic annotation substantially, since reading is considerably faster than doing linguistic annotation by hand. Our results show that gaze features do discriminate between most pairs of syntactic categories, and we show how we can use this to annotate words with part of speech across domains, when tag dictionaries enable us to narrow down the set of potential categories.
\end{abstract}

\section{Introduction}

Eye movements during reading is a wellestablished proxy for cognitive processing, and it is well-known that readers are more likely to fixate on words from open syntactic categories (verbs, nouns, adjectives) than on closed category items like prepositions and conjunctions (Rayner, 1998; Nilsson and Nivre, 2009). Generally, readers seem to be most likely to fixate and re-fixate on nouns (Furtner et al., 2009). If reading behavior is affected by syntactic category, maybe reading behavior can, conversely, also tell us about the syntax of words in context.

This paper investigates to what extent gaze data can be used to predict syntactic categories. We show that gaze data can effectively be used to discriminate between a wide range of part of speech
(POS) pairs, and gaze data can therefore be used to significantly improve type-constrained POS taggers. This is potentially useful, since eye-tracking data becomes more and more readily available with the emergence of eye trackers in mainstream consumer products (San Agustin et al., 2010). With the development of robust eye-tracking in laptops, it is easy to imagine digital text providers storing gaze data, which could then be used to improve automated analysis of their publications.

Contributions We are, to the best of our knowledge, the first to study reading behavior of syntactically annotated, natural text across domains, and how gaze correlates with a complete set of syntactic categories. We use logistic regression to show that gaze features discriminate between POS pairs, even across domains. We then show how gaze features can improve a cross-domain supervised POS tagger. We show that gaze-based predictions are robust, not only across domains, but also across subjects.

\section{Experiment}

In our experiment, 10 subjects read syntactically annotated sentences from five domains.

Data The data consists of 250 sentences: 50 sentences (min. 3 tokens, max. 120 characters), randomly sampled from each of five different, manually annotated corpora: Wall Street Journal articles (WSJ), Wall Street Journal headlines (HDL), emails (MAI), weblogs (WBL), and Twitter (TWI). WSJ and HDL syntactically annotated sentences come from the OntoNotes 4.0 release of the English Penn Treebank. ${ }^{1}$ The MAI and WBL sections come from the English Web Treebank. ${ }^{2}$

\footnotetext{
${ }^{1}$ catalog. Idc. upenn. edu/LDC2011T03

${ }^{2}$ catalog.ldc.upenn. edu/LDC2012T13
} 

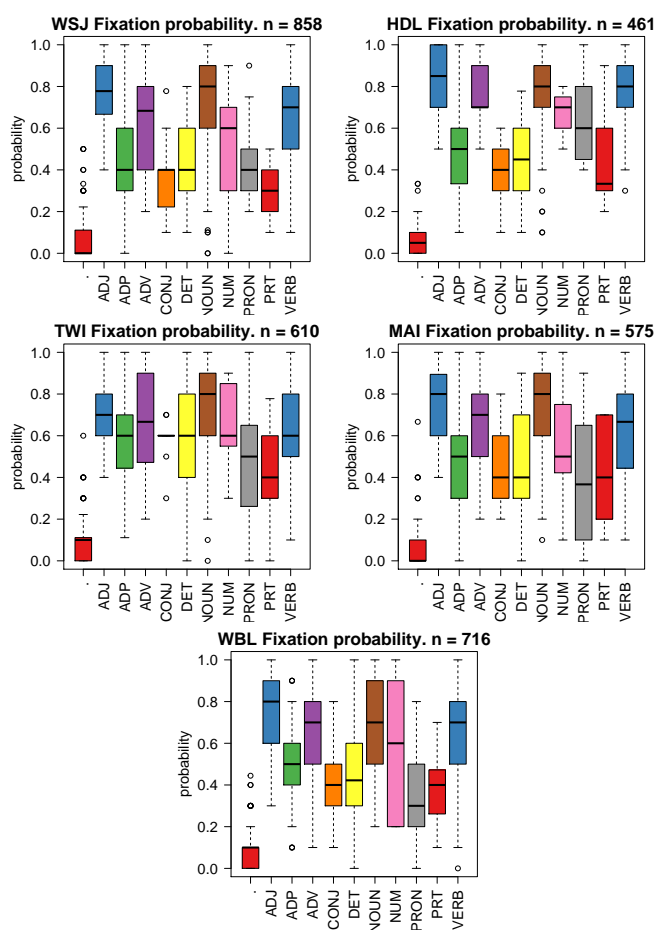

Figure 1: Fixation probability boxplots across five domains

The TWI data comes from the work of Foster et al. (2011). We mapped the gold labels to the 12 Universal POS (Petrov et al., 2011), but discarded the category $\mathrm{X}$ due to data sparsity.

Experimental design The 250 items were read by all 10 participants, but participants read the items in one of five randomized orders. Neither the source domain for the sentence, nor the POS tags were revealed to the participant at any time. One sentence was presented at a time in black on a light gray background. Font face was Verdana and font size was 25 pixels. Sentences were centered vertically, and all sentences could fit into one line. All sentences were preceded by a fixation cross. The experiment was self-paced. To switch to a new sentence and to ensure that the sentence was actually processed by the participant, participants rated the immediate interest towards the sentence on a scale from 1-6 by pressing the corresponding number on the numeric keypad. Participants were instructed to read and continue to the next sentence as quickly as possible. The actual experiment was preceded by 25 practice sentences to familiarize the participant with the experimental setup.

Our apparatus was a Tobii X120 eye tracker with a 15" monitor. Sampling rate was $120 \mathrm{~Hz}$ binocular. Participants were seated on a chair approximately $65 \mathrm{~cm}$ from the display. We recruited
10 participants ( 7 male, mean age $31.30 \pm 4.74)$ ) from campus. All were native English speakers. Their vision was normal or corrected to normal, and none were diagnosed with dyslexia. All were skilled readers. Minimum educational level was an ongoing MA. Each session lasted around 40 minutes. One participant had no fixations on a few sentences. We believe that erroneous key strokes caused the participant to skip a few sentences.

Features There are many different features for exploring cognitive load during reading (Rayner, 1998). We extracted a broad selection of cognitive effort features from the raw eye-tracking data in order to determine which are more fit for the task. The features are inspired by Salojärvi et al. (2003), who used a similarly exploratory approach. We wanted to cover both oculomotor features, such as fixations on previous and subsequent words, and measures relating to early (e.g. first fixation duration) and late processing (e.g. regression destinations / departure points and total fixation time). We also included reading speed and reading depth features, such as fixation probability and total fixation time per word. In total, we have 32 gaze features, where some are highly correlated (such as number of fixations on a word and total fixation time per sentence).

Dundee Corpus The main weakness of the experiment is the small dataset. As future work, we plan to replicate the experiment with a $\$ 99$ eye tracker for subjects to use at home. This will make it easy to collect thousands of sentences, leading to more robust gaze-based POS models. Here, instead, we include an experiment with the Dundee corpus (Kennedy and Pynte, 2005). The Dundee corpus is a widely used dataset in research on reading and consists of gaze data for 10 subjects reading 20 newswire articles (about 51,000 words). We extracted the same word-based features as above, except probability for 1 st and 2nd fixation, and sentence-level features (in the Dundee corpus, subjects are exposed to multiple sentences per screen window), and used them as features in our POS tagging experiments (§3).

Learning experiments In our experiments, we used type-constrained logistic regression with L2-regularization and type-constrained (averaged) structured perceptron (Collins, 2002; Täckström et al., 2013). In all experiments, unless otherwise stated, we trained our models on four domains and evaluated on the fifth to avoid over-fitting to the 


\begin{tabular}{c|c|c}
\hline Rank & Feature & \% of votes \\
\hline 0 & Fixation prob & 19.0 \\
1 & Previous word fixated binary & 13.7 \\
2 & Next word fixated binary & 13.2 \\
3 & nFixations & 12.2 \\
4 & First fixation duration on every word & 9.1 \\
5 & Previous fixation duration & 7.0 \\
6 & Mean fixation duration per word & 6.6 \\
7 & Re-read prob & 5.7 \\
8 & Next fixation duration & 2.0 \\
9 & Total fixation duration per word & 2.0 \\
\hline
\end{tabular}

Table 1: 10 most used features by stability selection from logistic regression classification of all POS pairs on all domains, 5-fold cross validation.

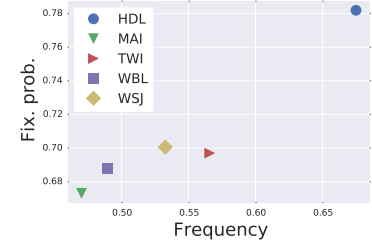

(a) Content words

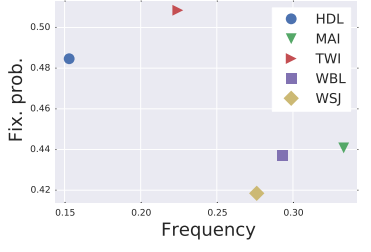

(b) Function words
Figure 2: Scatter plot of frequency and fixation probability for content words (NOUN, VERB, ADJ, NUM) and function words (PRON, CONJ, ADP, DET, PRT)

characteristics of a specific domain. Our tag dictionary is from Wiktionary ${ }^{3}$ and covers $95 \%$ of all tokens.

\section{Results}

Domain differences Our first observation is that the gaze characteristics differ slightly across domains, but more across POS. Figure 1 presents the

\footnotetext{
${ }^{3}$ https:// code.google.com/p/ wikily-supervised-pos-tagger/downloads/ list
}

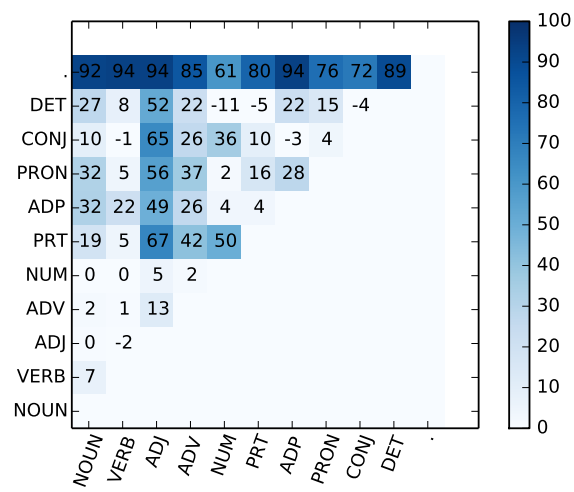

Figure 3: Error reduction of logistic regression over a majority baseline. All domains fixation probabilities across the 11 parts of speech. While the overall pattern is similar across the five domains (open category items are more likely to be fixated), we see domain differences. For example, pronouns are more likely to be fixated in headlines. The explanation could lie in the different distributions of function words and content words. It is established and unchallenged that function words are fixated on about $35 \%$ of the time and content words are fixated on about $85 \%$ of the time (Rayner and Duffy, 1988). In our data, these numbers vary among the domains according to frequency of that word class, see Figure 2. Figure 2a shows that there is a strong linear correlation between content word frequency and content word fixation probability among the different domains: Pearson's $\rho=0.909$. From Figure 2b, there is a negative correlation between function word frequency and function word fixation probability: Pearson's $\rho=-0.702$.

Predictive gaze features To investigate which gaze features were more predictive of part of speech, we used stability selection (Meinshausen and Bühlmann, 2010) with logistic regression classification on all binary POS classifications. Fixation probability was the most informative feature, but also whether the words around the word is fixated is important along with number of fixations. In our binary discrimination and POS tagging experiments, using L2-regularization or averaging with all features was superior (on Twitter data) to using stability selection for feature selection. We also asked a psycholinguist to select a small set of relatively independent gaze features fit for the task (first fixation duration, fixation probability and re-read probability), but again, using all features with L2-regularization led to better performance on the Twitter data.

Binary discrimination First, we trained L2regularized logistic regression models to discriminate between all pairs of POS tags only using gaze features. In other words, for example we selected all words annotated as NOUN or VERB, and trained a logistic regression model to discriminate between the two in a five-fold cross validation setup. We report error reduction $\frac{\text { acc-baseline }}{1-\text { baseline }}$ in Figure 3.

POS tagging We also tried evaluating our gaze features directly in a supervised POS tagger. ${ }^{4} \mathrm{We}$

\footnotetext{
${ }^{4}$ https://github.com/coastalcph/ rungsted
} 


\begin{tabular}{c|ccccc}
\hline & SP & +GAZE & +DGAZE & +FREQLEN & +DGAZE+FREQLEN \\
\hline HDL & 0.807 & 0.822 & 0.822 & 0.826 & $\mathbf{0 . 8 4 3}$ \\
MAI & 0.791 & 0.831 & $\mathbf{0 . 8 3 4}$ & 0.795 & 0.831 \\
TWI & 0.771 & 0.787 & $\mathbf{0 . 8 0 0}$ & 0.772 & 0.793 \\
WBL & 0.836 & 0.854 & 0.858 & 0.850 & $\mathbf{0 . 8 6 1}$ \\
WSJ & 0.831 & 0.837 & 0.838 & 0.831 & $\mathbf{0 . 8 5 9}$ \\
\hline Macro-av & 0.807 & 0.826 & 0.830 & 0.815 & $\mathbf{0 . 8 3 7}$
\end{tabular}

Table 2: POS tagging results on different test sets using 200 out-of-domain sentences for training. DGAZE is using gaze features from Dundee. Best result for each row in bold face

trained a type-constrained (averaged) perceptron model with drop-out and a standard feature model (from Owoputi et al. (2013)) augmented with the above gaze features. The POS tagger was trained on a very small seed of data (200 sentences), doing 20 passes over the data, and evaluated on out-ofdomain test data; training on four domains, testing on one. For the gaze features, instead of using token gaze features, we first built a lexicon with average word type statistics from the training data. We normalize the gaze matrix by dividing with its standard deviation. This is the normalizer in Turian et al. (2010) with $\sigma=1.0$. We condition on the gaze features of the current word, only. We compare performance using gaze features to using only word frequency, estimating from the (unlabeled) English Web Treebank corpus, and word length (FREQLEN).

The first three columns in Table 2 show, that gaze features help POS tagging, at least when trained on very small seeds of data. Error reduction using gaze features from the Dundee corpus (DGAZE) is $12 \%$. We know that gaze features correlate with word frequency and word length, but using these features directly leads to much smaller performance gains. Concatenating the two features sets leads to the best performance, with an error reduction of $16 \%$.

In follow-up experiments, we observe that averaging over 10 subjects when collecting gaze features does not seem as important as we expected. Tagging accuracies on raw (non-averaged) data are only about $1 \%$ lower. Finally, we also tried running logistic regression experiments across subjects rather than domains. Here, tagging accuracies were again comparable to our set-up, suggesting that gaze features are also robust across subjects.

\section{Related work}

Matthies and Søgaard (2013) present results that suggest that individual variation among (academically trained) subjects' reading behavior was not a greater source of error than variation within subjects, showing that it is possible to predict fixations across readers. Our work relates to such work, studying the robustness of reading models across domains and readers, but it also relates in spirit to research on using weak supervision in NLP, e.g., work on using HTML markup to improve dependency parsers (Spitkovsky, 2013) or using clickthrough data to improve POS taggers (Ganchev et al., 2012).

\section{Conclusions}

We have shown that it is possible to use gaze features to discriminate between many POS pairs across domains, even with only a small dataset and a small set of subjects. We also showed that gaze features can improve the performance of a POS tagger trained on small seeds of data.

\section{References}

Michael Collins. 2002. Discriminative training methods for Hidden Markov Models. In EMNLP.

Jennifer Foster, Ozlem Cetinoglu, Joachim Wagner, Josef Le Roux, Joakim Nivre, Deirde Hogan, and Josef van Genabith. 2011. From news to comments: Resources and benchmarks for parsing the language of Web 2.0. In IJCNLP.

Marco R Furtner, John F Rauthmann, and Pierre Sachse. 2009. Nomen est omen: Investigating the dominance of nouns in word comprehension with eye movement analyses. Advances in Cognitive Psychology, 5:91.

Kuzman Ganchev, Keith Hall, Ryan McDonald, and Slav Petrov. 2012. Using search-logs to improve query tagging. In $A C L$. 
Alan Kennedy and Joël Pynte. 2005. Parafoveal-onfoveal effects in normal reading. Vision research, 45(2):153-168.

Franz Matthies and Anders Søgaard. 2013. With blinkers on: Robust prediction of eye movements across readers. In EMNLP, Seattle, Washington, USA.

Nicolai Meinshausen and Peter Bühlmann. 2010. Stability selection. Journal of the Royal Statistical Society: Series B (Statistical Methodology), 72(4):417-473.

Matthias Nilsson and Joakim Nivre. 2009. Learning where to look: Modeling eye movements in reading. In $C o N L L$.

Olutobi Owoputi, Brendan O'Connor, Chris Dyer, Kevin Gimpel, Nathan Schneider, and Noah A Smith. 2013. Improved part-of-speech tagging for online conversational text with word clusters. In NAACL.

Slav Petrov, Dipanjan Das, and Ryan McDonald. 2011. A universal part-of-speech tagset. arXiv preprint arXiv:1104.2086.

K.; Rayner and S. A. Duffy. 1988. On-line comprehension processes and eye movements in reading. In G. E. MacKinnon M. Daneman and T. G. Waller, editors, Reading research: Advances in theory and practice, pages 13-66. Academic Press, New York.

Keith Rayner. 1998. Eye movements in reading and information processing: 20 years of research. Psychological bulletin, 124(3):372.

Jarkko Salojärvi, Ilpo Kojo, Jaana Simola, and Samuel Kaski. 2003. Can relevance be inferred from eye movements in information retrieval. In Proceedings of WSOM, volume 3, pages 261-266.

Javier San Agustin, Henrik Skovsgaard, Emilie Mollenbach, Maria Barret, Martin Tall, Dan Witzner Hansen, and John Paulin Hansen. 2010. Evaluation of a low-cost open-source gaze tracker. In Proceedings of the 2010 Symposium on Eye-Tracking Research \& Applications, pages 77-80. ACM.

Valentin Ilyich Spitkovsky. 2013. Grammar Induction and Parsing with Dependency-and-Boundary Models. Ph.D. thesis, STANFORD UNIVERSITY.

Oscar Täckström, Dipanjan Das, Slav Petrov, Ryan McDonald, and Joakim Nivre. 2013. Token and type constraints for cross-lingual part-of-speech tagging. TACL, 1:1-12.

Joseph Turian, Lev Ratinov, and Yoshua Bengio. 2010. Word representations: a simple and general method for semi-supervised learning. In $A C L$. 